\title{
Nachwuchsmangel und Interprofessionalität
}

\author{
An der 60. Konsultativtagung der deutschsprachigen Ärzteorganisationen vom 3. bis 5 . \\ Juli 2014 kamen rund 35 Expertinnen und Experten in Brunnen zusammen. Am Vier- \\ waldstättersee tobte ein heftiger Föhnsturm und angeregt diskutiert wurde auch im Ple- \\ narsaal. Zu den brennenden Themen wie Nachwuchsmangel, Interprofessionalität oder \\ Technologien als Antwort auf den Ärztemangel herrschte nicht immer Einigkeit, aber \\ doch war der Wille da, die anstehenden Herausforderungen gemeinsam anzupacken.
}

Dr. med. Jürg Schlup, Präsident der FMH, eröffnete die Tagung mit einer kurzen Übersicht über die Gesundheitssysteme der teilnehmenden Länder Schweiz, Deutschland, Österreich, Italien (Südtirol) und Luxemburg. In allen diesen Ländern befinden sich die Systeme auf einem hohen Niveau (hohe Lebenserwartung, gute Bewertung durch Bevölkerung, kurze Wartezeiten auf Termin bei Spezialarzt) und die Versorgung ist momentan gut. Aber die Bevölkerung wird immer älter, einige dieser Länder bilden zu wenig Ärzte aus und der Anteil von Ärztinnen und Ärzten, die in den nächsten Jahren das Pensionsalter erreichen, nimmt zu - es besteht ein klares Nachwuchsproblem.

\section{Nachwuchsmangel bei den Ärzten}

Eine Übersicht über den Nachwuchsmangel gab Dr. med. Max Kaplan, Präsident der Bayerischen Ärztekammer. Die Vertreter der einzelnen Länder präsentierten anschliessend die jeweilige Datenlage (Tab. 1). Prinzipiell gibt es drei wichtige Gründe für den Ärztemangel. Erstens bewirkt die demographische Entwicklung, dass der Bedarf für medizinische Leistungen steigt. Zweitens verlässt die Generation der Baby- boomer in den nächsten Jahren altersbedingt die kurative Tätigkeit. Drittens arbeiten die jungen Ärztinnen und Ärzte insgesamt öfter Teilzeit als frühere Generationen. Das heisst: Mehr Ärzte leisten nicht notwendigerweise mehr Arbeit.

Obwohl immer mehr junge Leute Medizin studieren, ist die Abwanderungsrate bei den jungen Ärztinnen und Ärzten hoch. Wichtige Gründe für den Ausstieg sind unter anderem Hierarchie und autoritäre Führung in den Spitälern, hohe zeitliche Belastung resp. Unvereinbarkeit von Familie und Beruf, die als nicht leistungsgerecht empfundene Vergütung und zu viele nicht-ärztliche Tätigkeiten. Max Kaplan präsentierte Lösungsansätze. So sollten die Arbeitsbedingungen in den Kliniken verändert werden, zum Beispiel mit flexibler Arbeitszeitgestaltung, Kinderkrippen, flachen Hierarchien usw. Auch die Niederlassung muss attraktiver werden, etwa mittels eines niederschwelligen Praxis-Einstiegs in Anstellung, neue Versorgungsformen, Neustrukturierung des Notfalldienstes, Stärkung der ländlichen Regionen usw. Ausserdem gilt es, die Arbeitsteilung innerhalb der Gesundheitsberufe neu zu überdenken und zu definieren.
Korrespondenz:

FMH/Abteilung Kommunikation Elfenstrasse 18

CH-3000 Bern Tel. 0313591111 Fax 0313591112

kommunikation[at]fmh.ch

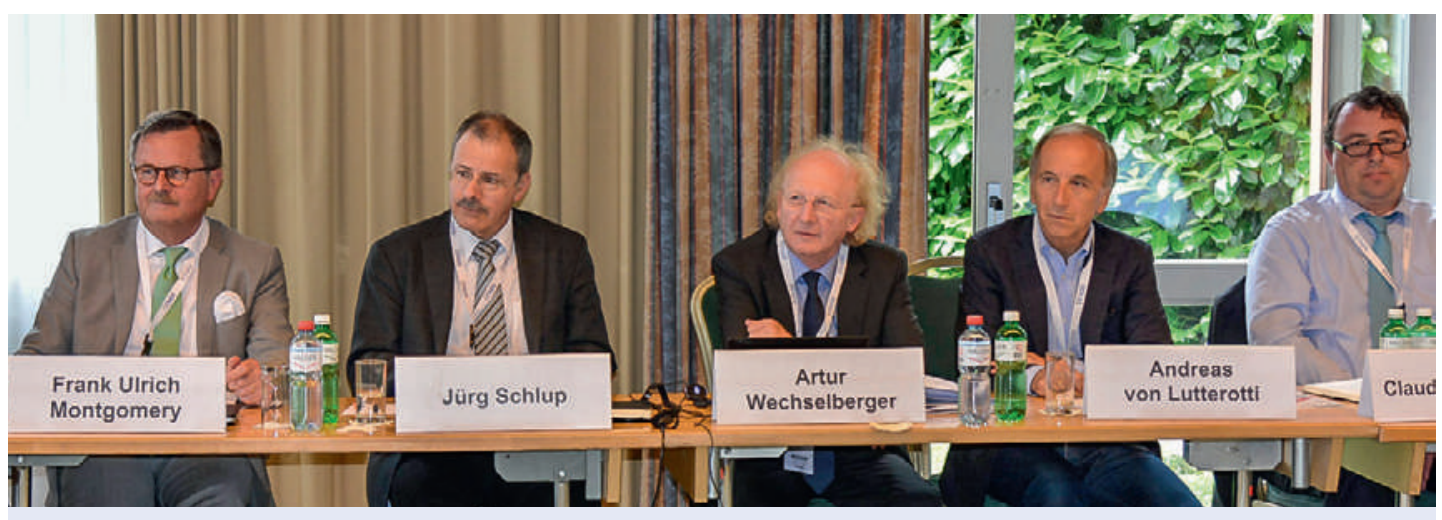

Fünf Länder, eine gemeinsame Sprache - die Präsidenten der deutschsprachigen Ärzteorganisationen.

Von links nach rechts: Frank Ulrich Montgomery (Deutschland), Jürg Schlup (Schweiz), Artur Wechselberger (Österreich), Andreas von Lutterotti (Südtirol) und Claude Schummer (Luxemburg). 
Tabelle 1

Nachwuchsmangel - Fakten aus verschiedenen Ländern.

$\begin{array}{ll}\text { Schweiz } & \text { Aufnahmekapazität der Schweizer Unis für Medizinstudierende: } 1600 \text { pro Jahr. } \\ \text { Jährlich werden } 800 \text { Arztdiplome erteilt. } \\ \text { Ausländische Ärzte ersetzen die fehlenden Ärzte aus dem Inland. } \\ 2013 \text { hatten } 29 \% \text { der tätigen Ärzte ein ausländisches Diplom (2003: 17\%); im stationären Sektor 36\% (2003: 24\%), } \\ \text { im ambulanten Sektor 23\% (2003: 11\%). } \\ \text { Hoher Anteil von Privatärzten, die keine Kassenverträge haben wollen. } \\ \text { Der Anteil an ausländischen Medizinstudierenden ist auf 25\% beschränkt, beim Abschluss sind es rund 30\%. } \\ \text { Österreich } \\ \text { Östen diplomierten Ärzten werden 50\% nicht in der Versorgung tätig (Ausländer gehen in ihr Heimatland zurück, } \\ \text { Keine Ausbildung von Ärztinnen und Ärzten, die Studierenden müssen nach dem ersten Studienjahr } \\ \text { ins Ausland (28\% Deutschland, 23\% Belgien, 23\% Frankreich). } \\ \text { Eine Medical School mit rund 100 Studienplätzen pro Jahr ist geplant. } \\ \text { Luxemburg hat eine geringe Ärztedichte und im europäischen Vergleich sehr wenig Allgemeinmediziner, } \\ \text { Luxemburg ein gut funktionierendes Gesundheitssystem, da die Ärztinnen und Ärzte viel arbeiten. } \\ \text { Das Land ist vor allem bei den Spezialärzten stark vom Ausland abhängig. } \\ \text { Im Südtirol ist es schwierig, Ärztinnen und Ärzte aus dem Ausland zu rekrutieren, weil die Bewerber Italienisch und Deutsch } \\ \text { sprechen müssen (mit Nachweis der Kompetenz resp. Sprachprüfung). } \\ \text { Für Südtiroler Studienbewerber wird die Situation zunehmend schwieriger: Nur wenige studieren im restlichen Italien, } \\ \text { weil die Zulassung zum Studium dort sehr streng ist und sie einen Sprachnachteil haben (Italienisch ist nicht ihre Muttersprache). } \\ \text { Die meisten gehen nach Österreich, aber dort sind 75\% der Studienplätze den Einheimischen vorbehalten. }\end{array}$

Einige Voten aus der nachfolgenden, lebhaften Diskussion:

- «Heute muss man de facto 1,7-1,8 Studierende ausbilden, um eine 100\%-Stelle zu besetzen. Daher sagen Studierendenzahlen allein nichts darüber aus, wie die spätere Versorgung sein wird.»

- «Im stationären Bereich arbeiten Frauen gleich viel wie Männer, im ambulanten Bereich aber nur einen Drittel so viel. Dies liegt häufig am Notfalldienst, der sich schwer mit dem Familienleben vereinbaren lässt.»

- «Die jungen Ärztinnen und Ärzte sind engagiert und wollen arbeiten, aber sie möchten diese Arbeit planen können. Es ist wichtig, die entsprechenden Strukturen zu schaffen.»

\section{Interprofessionalität und Task shifting}

Dr. iur. Lukas Stärker und Dr. med. Harald Mayer, Österreichische Ärztekammer, berichteten zum Thema Interprofessionalität von der Situation in Österreich. Dort können die Pflegenden im pflegerischen Tätigkeitsbereich ohne ärztliche Anordnung arbeiten. «Beim Task shifting sehen wir zwei Problembereiche», meinte Lukas Stärker. «Zum einen werden die Berufsbilder bei den Pflegenden nicht vollständig ausgeübt, zum anderen gehört die Pflege in Österreich zu den Mangelberufen.»

Zwischen Oktober 2013 und Januar 2014 führte die Österreichische Ärztekammer in verschiedenen europäischen Ländern eine Umfrage durch, um vertiefte Kenntnisse zur Ausbildung und zum Tätigkeitsumfang von allgemeinen Pflegekräften zu erlangen.
Antworten kamen aus Belgien, Deutschland, Frankreich, Island, Italien, Litauen, Niederlande, Polen, Schweiz, Slowakei, Slowenien, Spanien und Tschechien. Dabei zeigten sich grosse Unterschiede zwischen den 13 Ländern. Am meisten Eigenverantwortung haben die Pflegenden in Frankreich, Island und Polen, wo sie beispielsweise Infusionen und Injektionen in Entscheidungsautonomie verabreichen dürfen. Im Allgemeinen liegt aber die Anordnungsverantwortung beim Arzt, die Durchführungsverantwortung beim Pflegepersonal.

Mehr als die Hälfte der befragten Länder berichtete über Pläne, das Qualifikationsniveau der Pflegenden anzuheben und ihren Tätigkeitsumfang auszuweiten. Eine besonders grosse Vielfalt an Spezialisierungen der Pflegenden kennen Belgien, Tschechien und die Niederlande, wo ein «Master in Advanced Nursing Practice» in fünf Fachgebieten erworben werden kann. Bei der Akademisierung der Pflegeberufe sahen die Referenten die Schwierigkeit, dass akademisch ausgebildete Pflegende häufig nicht mehr in die praktische Pflege zurückkehren wollen. Für sie ist eine Verbesserung der Ausbildung, mit dem Ziel einer effektiven und effizienten Versorgungsstruktur, unter folgenden Voraussetzungen sinnvoll: Einerseits muss die Ausbildung bedarfsund zukunftsorientiert sowie mit anderen Berufsgruppen abgestimmt sein. Andererseits gilt es auch, die Bedürfnisse der jeweiligen Berufsgruppe vorab zu klären und die rechtlichen Rahmenbedingungen und Kompetenzen klar zu regeln. 
Pflegesituation in der Schweiz und anderswo

Im Anschluss stellten die Ländervertreter die Situation der Pflegenden in ihren Regionen vor. In der Schweiz herrscht auch beim Pflegepersonal ein deutlicher Nachwuchsmangel. «Gemäss dem nationalen Versorgungsbericht für die Gesundheitsberufe 2009 werden bis im Jahr 2020 rund 5000 Pflegekräfte zu wenig ausgebildet», sagte Esther Kraft, Leiterin der

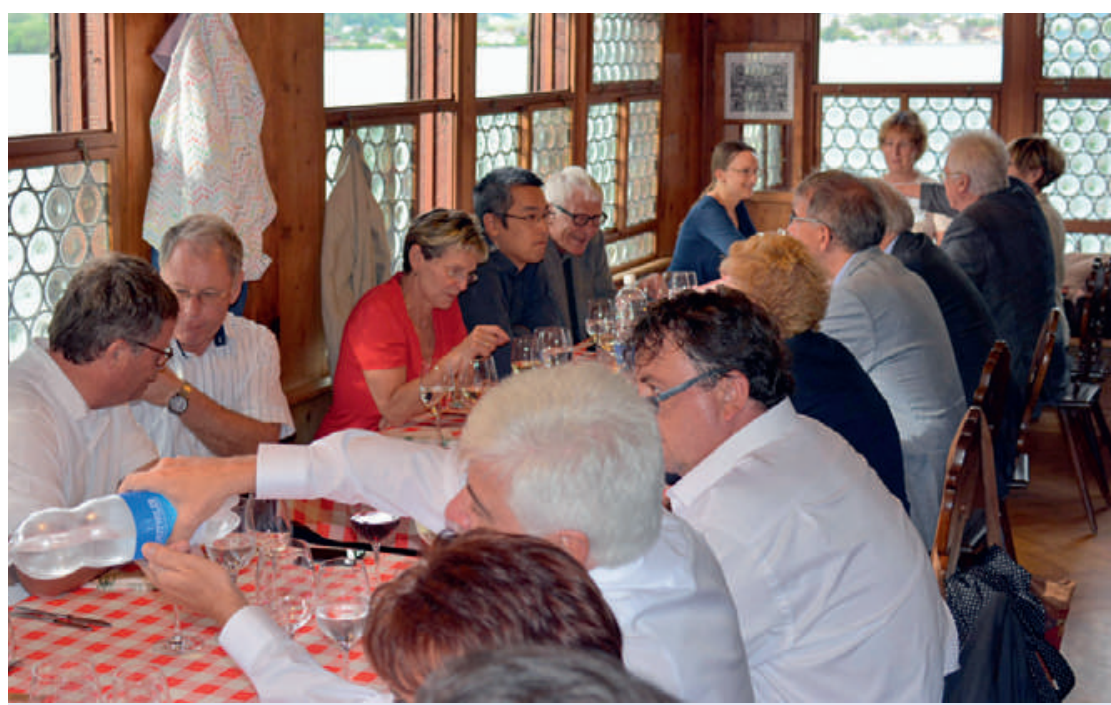

Auch nach Abschluss der «offiziellen» Diskussionen im Plenarsaal tauschen sich die Teilnehmenden beim Abendessen aus.

\section{Fazit der Diskussionen}

- Organisierte Sterbehilfe: Die Beihilfe zum Suizid gehört nicht zu den ärztlichen Aufgaben und darf keinesfalls zu einer Berufspflicht werden. Im Sinne einer aktiven Suizidprävention bedeutet ärztliche Verantwortung und ärztliches Handeln, den Patienten palliativmedizinische Angebote zu machen.

- Technologien als Antwort auf Ärztemangel: Neue Technologien ersetzen sicher nicht den Arzt und die Ärztin. Obwohl sie durchaus ressourcenschonend sind, sollte man nicht zu hohe Erwartungen darin setzen.

- Nachwuchsmangel: Für den sich in allen deutschsprachigen Ländern abzeichnenden Ärztemangel sind verschiedene Faktoren verantwortlich: die demographische Entwicklung der Bevölkerung und der Ärzteschaft, der Fortschritt der medizinisch-technischen Entwicklung und der Verbleib in tradierten Organisationsstrukturen, die nicht den Vorstellungen der jungen Ärztegeneration entsprechen, anstatt eine effiziente medizinische Versorgung in den Fokus zu stellen. Für ihre Qualität ist nicht allein die Anzahl Ärztinnen und Ärzte entscheidend, die Verbesserung der Strukturen und die Arbeitsteilung innerhalb der Gesundheitsberufe sind ebenso wichtig.

- Interprofessionalität im Gesundheitswesen/Task shifting: Pflegekräfte sollen medizinische, diagnostisch-therapeutische Tätigkeiten einzig auf ärztliche Anordnung durchführen. Aus der ärztlichen Prozessverantwortung folgt die Notwendigkeit der ärztlichen Mitsprache bei der Entwicklung der Ausbildungscurricula für alle Gesundheitsberufe.

- Ist der Arztberuf noch ein freier Beruf?: Ethik, Kompetenz und Freiheit sind Wesensmerkmale der ärztlichen Berufsausübung. Sie sind das Fundament der beruflichen Selbstverpflichtung und dienen dem Patientenwohl. Jede Ärztegeneration fühlt sich verpflichtet, diese vorzuleben und an die nächste Generation weiterzugeben.
Abteilung Daten, Demographie und Qualität der FMH. Schon heute haben $27 \%$ der Pflegefachpersonen in Alters- und Pflegeheimen ein ausländisches Diplom (Genf: 70,4\%, Appenzell Innerrhoden: <5\%). Die Zufriedenheit der Pflegekräfte in der Schweiz ist aber (noch) recht hoch: Mindestens 90\% sind mit ihrem Beruf «eher zufrieden» oder «sehr zufrieden», und über $80 \%$ äussern sich positiv über ihre Arbeitsstelle. Ein aktueller Bericht des Gesundheitsobservatoriums Obsan zeigt auf, dass in der Schweiz eine Pflegekraft durchschnittlich acht Patienten betreut (in Norwegen fünf, in Deutschland 13 Patienten). In der Schweiz beabsichtigen 28\% der Pflegenden sich nächstens eine neue Stelle zu suchen, in Deutschland sind es $44 \%$.

Wie gross die Unterschiede bei den Kompetenzen des Pflegepersonals sind, zeigen zwei Beispiele der Vertreter aus dem Südtirol und Luxemburg: Im Südtirol (Italien) ist die Pflege ein dreijähriges Hochschulstudium mit Doktorat. Vom Titel her sind Pflegefachpersonen nicht mehr von Ärzten zu unterscheiden. Mehrere Versuche, die Kompetenzen der Pflegenden auszuweiten, wie beispielsweise die Triagierung der Patienten auf der Notfallstation, wurden aufgrund der unklaren Verantwortungsbereiche von der Südtiroler Ärztekammer gestoppt. In Luxemburg hingegen gibt es die «am besten bezahlten Pfleger der Welt» - mit der Folge, dass in den Nachbarländern Frankreich und Belgien in den grenznahen Gebieten «Pflegewüsten» entstanden sind. Die Pflegenden in Luxemburg haben aber auch viele Kompetenzen, zum Beispiel in der Anästhesie: Dort ist ein Anästhesist gleichzeitig für fünf Operationen verantwortlich, die Hauptarbeit am Patienten leistet daher eine Pflegefachkraft.

\section{Technologie als Lösung für den Ärztemangel?} Am Nachmittag teilten sich die Tagungsteilnehmer in fünf Gruppen auf, um in Workshops zu verschiedenen Themen zu diskutieren (s. Kasten). Im Workshop «Technologien als Antwort auf Ärztemangel?» waren zwar alle Teilnehmenden überzeugt, dass Technologien wie e-Health, Telemedizin, portable Diagnosegeräte oder mobile Arztpraxen kommen werden. Der Nutzen und die Sinnhaftigkeit dieser Entwicklung wurden dennoch unterschiedlich gewertet. Einig war man sich, dass die neuen Technologien den Arzt nicht ersetzen werden. Sie können aber vor allem dort hilfreich sein, wo sie in der Menge eine Entlastung bringen, beispielsweise bei Patienten mit häufigen Erkrankungen wie Diabetes, COPD oder Herzinsuffizienz. Strukturelle und personelle Probleme wird die Technik allein kaum beheben können, denn parallel zu den technischen Entwicklungen wächst das medizinische Wissen - und die Bedürfnisse an die medizinische Versorgung steigen.

Quelle: 60. Konsultativtagung der deutschsprachigen Ärzteorganisationen, Brunnen, 3.-5. Juli 2014. 